\title{
Pacific
}

Journal of

Mathematics

MULTIPLIERS BETWEEN INVARIANT SUBSPACES OF THE BACKWARD SHIFT

ROBERT BRUCE CROFOOT

Volume $166 \quad$ No. 2

December 1994 


\section{MULTIPLIERS BETWEEN INVARIANT SUBSPACES OF THE BACKWARD SHIFT}

\section{R. BRUCE CROFOOT}

Contained in the Hardy space $H^{2}$ on the unit disk in the complex plane are certain Hilbert spaces which are invariant under the adjoint of the shift. One such space $\mathcal{H}(b)$ is associated with each function $b$ in the closed unit ball of $H^{\infty}$. In the special case where $b$ is an inner function, $\mathcal{H}(b)$ is just the subspace of $H^{2}$ orthogonal to the shift-invariant subspace $b H^{2}$. It is proven here that for any functions $b_{1}$ and $b_{2}$ in the closed ball of $H^{\infty}$, the spaces $\mathcal{H}\left(b_{1}\right)$ and $\mathcal{H}\left(b_{2}\right)$ are isometrically isomorphic under a multiplication operator if and only if there is a disk automorphism $\tau$ such that $b_{2}=\tau \circ b_{1}$. In this case, the multiplicative isomorphism is determined explicitly and uniquely. This motivates an investigation of multipliers between $\mathcal{H}\left(b_{1}\right)$ and $\mathcal{H}\left(b_{2}\right)$, that is, multiplication operators acting bijectively but not necessarily isometrically. Restricting to the case where $b_{1}$ and $b_{2}$ are inner functions, it is shown that a multiplier between given spaces is unique up to multiplication by a nonzero constant, and several theorems are proven concerning the existence of such multipliers. Finally, consideration is given to the implications of these results for the characterization of the invariant subspaces in $H^{2}$ on an annulus.

1. Introduction. To any analytic function $f$ on the unit disk can be applied the mappings $f \mapsto z f$ and $f \mapsto z^{-1}[f-f(0)]$. On the Hilbert space $H^{2}$ these mappings are adjoint operators called, respectively, the shift $S$ and the backward shift $S^{*}$. For convenience the notation $S$ and $S^{*}$ will be used even when the mappings are applied to functions not in $H^{2}$. If $f$ and $g$ are analytic functions on the disk, then $S^{*}(f g)=f S^{*} g+g(0) S^{*} f$. This simple algebraic identity will be applied repeatedly without comment. 
The shift is an example of a Toeplitz operator. In general, if $u \in L^{\infty}$, the Toeplitz operator with symbol $u$ is the bounded linear operator $T_{u}$ defined on functions $f$ in $H^{2}$ by $T_{u} f=P^{+}(u f)$, where $P^{+}$is the operator of orthogonal projection from $L^{2}$ onto $H^{2}$. (The Lebesgue spaces here are defined with respect to Lebesgue measure on the unit circle.) The adjoint of $T_{u}$ is $T_{u}^{*}=T_{\bar{u}}$. If $u$ is analytic, the projection $P^{+}$is redundant and $T_{u}$ reduces to multiplication by $u$. Note that $S=T_{z}$ and $S^{*}=T_{\bar{z}}$.

Occasionally it will be useful to allow $u$ to be unbounded. If $u \in L^{2}, T_{u}$ is defined as above except that now it is an unbounded operator with domain $\left\{f \in H^{2}: u f \in L^{2}\right\}$.

For any $h \in H^{2}, T_{h}$ commutes with $S$ and $T_{\bar{h}}$ commutes with $S^{*}$. The commutators of $T_{h}$ with $S^{*}$ and $T_{\bar{h}}$ with $S$ are rank-one operators:

$$
\begin{aligned}
S^{*} T_{h}-T_{h} S^{*} & =\left(S^{*} h\right) \otimes 1, \\
T_{\bar{h}} S-S T_{\bar{h}} & =1 \otimes\left(S^{*} h\right) .
\end{aligned}
$$

(If $f_{1}$ and $f_{2}$ are in $H^{2}, f_{1} \otimes f_{2}$ denotes the rank-one operator which acts on $h \in H^{2}$ according to the rule $f_{1} \otimes f_{2}(h)=\left\langle h, f_{2}\right\rangle f_{1}$, where $\langle\cdot, \cdot\rangle$ is the inner product in $H^{2}$.)

A subspace of $H^{2}$ will always mean a Hilbert subspace. As is well known, Beurling's theorm characterizes the nonzero $S$-invariant subspaces of $H^{2}$ as being precisely the subspaces $\phi H^{2}$, for inner functions $\phi$. The $S^{*}$-invariant subspaces are just the orthogonal complements of these. Thus there is associated with each inner function $\phi$ an $S^{*}$-invariant subspace, here denoted by $\mathcal{H}(\phi)$, which is the orthogonal complement of $\phi H^{2}$ in $H^{2}$. It is easy to show that $S^{*} \phi \in \mathcal{H}(\phi)$; in fact, $\mathcal{H}(\phi)$ is the $S^{*}$-invariant subspace generated by $S^{*} \phi$.

In $H^{2}$, evaluation of any function $f$ at a point $w$ of the disk is accomplished by the inner product $\left\langle f, k_{w}\right\rangle$, where $k_{w}=(1-$ $\bar{w} z)^{-1}$ is called the kernel for evaluation at $w$. The function $k_{w}$ is in $\mathcal{H}(\phi)$ if and only if $\phi(w)=0$. In particular, $1 \in \mathcal{H}(\phi)$ if and only if $\phi(0)=0$. If $\phi$ is a finite Blaschke product, then $\mathcal{H}(\phi)$ is finite-dimensional, with dimension equal to the number of zeros of $\phi$ (counting multiplicities); conversely, if $\mathcal{H}(\phi)$ is finite-dimensional, then $\phi$ is a finite Blaschke product [6, p. 33].

The $S^{*}$-invariant subspaces of $H^{2}$ can be placed in a more general 
context. Corresponding to any function $b$ in the closed unit ball of $H^{\infty}$ there is a Hilbert space $\mathcal{H}(b)$ contractively contained in $H^{2}$ (where "contractively" means that the $\mathcal{H}(b)$ norm dominates the $H^{2}$ norm). Introduced by de Branges and Rovnyak [1], these spaces have been investigated more recently by Sarason $[\mathbf{9}, \mathbf{1 0}]$. Sarason defines $\mathcal{H}(b)$ to be the range of the operator $\left(1-T_{b} T_{\bar{b}}\right)^{1 / 2}$, endowed with the Hilbert space structure which makes this operator into a partial isometry. For any $b, \mathcal{H}(b)$ is $S^{*}$-invariant $[\mathbf{9}$, p. 156]. In the special case where $b$ is an inner function, the operator $T_{b} T_{\bar{b}}$ is the orthogonal projection onto the subspace $b H^{2}$, and the Hilbert space $\mathcal{H}(b)$ is exactly the $S^{*}$-invariant subspace defined above, namely the subspace of $H^{2}$ orthogonal to $b H^{2}$.

In the Hilbert space $\mathcal{H}(b)$, the kernel for evaluation at $w$ is the function $k_{w}^{b}=[1-\overline{b(w)} b] k_{w}$. This means $\left\langle f, k_{w}^{b}\right\rangle_{b}=f(w)$ for all $f \in \mathcal{H}(b)$ and all points $w$ in the open unit disk. (The inner product and norm in $\mathcal{H}(b)$ will be distinguished notationally by a subscript b.)

The notion of a multiplier between two spaces $\mathcal{H}\left(b_{1}\right)$ and $\mathcal{H}\left(b_{2}\right)$ will be defined in Section 2. Of special interest are isometric multipliers, which are defined and characterized in Section 3. Beginning in Section 4, discussion is restricted to the subspaces $\mathcal{H}(\phi)$, where $\phi$ is an inner function. It is shown that if there exists a multiplier between two such subspaces, it is unique up to multiplication by a nonzero constant. In Section 5, some general theorems are proven concerning existence of multipliers and their relationship to the subspaces on which they act. Evaluation kernels act as multipliers in a simple manner, discussed in Section 6. Certain rational functions formed from these kernels are the only possible multipliers between finite dimensional subspaces. Section 7 describes how to construct multipliers as infinite products. In Section 8, multipliers are applied to certain invariant subspaces in $H^{2}$ on an annulus. Section 9 concludes with two open questions.

The unit disk will be denoted by $D$ and the unit circle by $T$. The symbols $N, Z, R$ and $C$ will be used respectively for the natural numbers, integers, real numbers and complex numbers. 


\section{Formulation of the Multiplier Problem.}

Definition 1. Let $b_{1}$ and $b_{2}$ be functions in the closed unit ball of $H^{\infty}$. A multiplier from $\mathcal{H}\left(b_{1}\right)$ onto $\mathcal{H}\left(b_{2}\right)$ is an analytic function $m$ on $D$ such that $m \mathcal{H}\left(b_{1}\right)=\mathcal{H}\left(b_{2}\right)$. A multiplier between $\mathcal{H}\left(b_{1}\right)$ and $\mathcal{H}\left(b_{2}\right)$ is an analytic function $m$ on $D$ such that $m \mathcal{H}\left(b_{1}\right)=\mathcal{H}\left(b_{2}\right)$ or $m \mathcal{H}\left(b_{2}\right)=\mathcal{H}\left(b_{1}\right)$.

For the remainder of this section, $b, b_{1}$ and $b_{2}$ will denote arbitrary functions in the closed unit ball of $H^{\infty}$ which are not unimodular constants (so that the corresponding spaces $\mathcal{H}(b), \mathcal{H}\left(b_{1}\right)$ and $\mathcal{H}\left(b_{2}\right)$ are nontrivial).

Suppose $m$ is a multiplier from $\mathcal{H}\left(b_{1}\right)$ onto $\mathcal{H}\left(b_{2}\right)$. Since $\mathcal{H}\left(b_{2}\right)$ contains outer functions (for example, the evaluation kernels), the functions in $\mathcal{H}\left(b_{2}\right)$ have no common inner factor and therefore no common zero. Consequently $m$ has no zeros, and $m^{-1}$ is an analytic function on $D$. Evidently $m$ is a multiplier from $\mathcal{H}\left(b_{1}\right)$ onto $\mathcal{H}\left(b_{2}\right)$ if and only if $m^{-1}$ is a multiplier from $\mathcal{H}\left(b_{2}\right)$ onto $\mathcal{H}\left(b_{1}\right)$.

Proposition 2. Let $m$ be an analytic function on $D$ with no zeros. The following statements are equivalent:

(i) $m \mathcal{H}(b)$ is $S^{*}$-invariant,

(ii) $S^{*} m \in m \mathcal{H}(b)$,

(iii) $S^{*}\left(m^{-1}\right) \in \mathcal{H}(b)$.

Proof. For any $f \in \mathcal{H}(b), S^{*}(m f)=m S^{*} f+f(0) S^{*} m$. From this identity and the $S^{*}$-invariance of $\mathcal{H}(b)$, it follows that (i) and (ii) are equivalent. The equivalence of (ii) and (iii) is a consequence of the identity $m(0) m S^{*}\left(m^{-1}\right)=-S^{*} m$.

Following immediately from this proposition are some useful necessary conditions for $m$ to be a multiplier:

COROllary 3. If $m$ is a multiplier from $\mathcal{H}\left(b_{1}\right)$ onto $\mathcal{H}\left(b_{2}\right)$, then $S^{*}\left(m^{-1}\right) \in \mathcal{H}\left(b_{1}\right)$ and $S^{*} m \in \mathcal{H}\left(b_{2}\right)$.

Corollary 4. If $m$ is a multiplier between $\mathcal{H}\left(b_{1}\right)$ and $\mathcal{H}\left(b_{2}\right)$, then $m$ and $m^{-1}$ are outer functions in $H^{2}$.

The following proposition shows that multipliers, when they exist, act as topological vector space isomorphisms. The proof is a standard application of the Closed Graph Theorem. 
Proposition 5. Let $m$ be a multiplier between $\mathcal{H}\left(b_{1}\right)$ and $\mathcal{H}\left(b_{2}\right)$. As an operator between $\mathcal{H}\left(b_{1}\right)$ and $\mathcal{H}\left(b_{2}\right)$, multiplication by $m$ is bounded. Its inverse, multiplication by $\mathrm{m}^{-1}$, is also bounded.

Proof. Suppose $\mathcal{H}\left(b_{2}\right)=m \mathcal{H}\left(b_{1}\right)$. Let $\left(f_{n}\right)_{n=1}^{\infty}$ be a convergent sequence in $\mathcal{H}\left(b_{1}\right)$ such that $\left(m f_{n}\right)_{n=1}^{\infty}$ converges in $\mathcal{H}\left(b_{2}\right)$. Let $f_{\infty}$ denote the limit of $\left(f_{n}\right)$, and let $g$ be the limit of $\left(m f_{n}\right)$. Since $f_{n} \rightarrow f_{\infty}$ in $\mathcal{H}\left(b_{1}\right)$ (hence also in $\left.H^{2}\right), f_{n} \rightarrow f_{\infty}$ pointwise. Thus, for all $z \in D, m(z) f_{n}(z) \longrightarrow m(z) f_{\infty}(z)$. On the other hand, since $m f_{n} \rightarrow g$ in $\mathcal{H}\left(b_{2}\right), m(z) f_{n}(z) \longrightarrow g(z)$ for all $z \in D$. Therefore $g=m f_{\infty}$. This proves that the operator of multiplication by $m$ from $\mathcal{H}\left(b_{1}\right)$ onto $\mathcal{H}\left(b_{2}\right)$ has a closed graph, which implies that it is bounded. The same argument applies to $m^{-1}$ as a multiplier from $\mathcal{H}\left(b_{2}\right)$ onto $\mathcal{H}\left(b_{1}\right)$.

3. Isometric multipliers. Some multipliers act to preserve not only the topology, but the norm. These will be called isometric multipliers. Here is a precise definition:

DEFINITION 6. Let $b_{1}$ and $b_{2}$ be functions in the closed unit ball of $H^{\infty}$. A multiplier $m$ from $\mathcal{H}\left(b_{1}\right)$ onto $\mathcal{H}\left(b_{2}\right)$ is called an isometric multiplier if multiplication by $m$ is an isometry on $\mathcal{H}\left(b_{1}\right)$.

We will prove (Theorem 10) that an isometric multiplier exists between spaces $\mathcal{H}\left(b_{1}\right)$ and $\mathcal{H}\left(b_{2}\right)$ if and only if $b_{2}=\tau \circ b_{1}$, where $\tau$ is some conformal automorphism of the disk. The isometry was suggested by a theorem of D. Sarason [11] (p. 489, Theorem 2), which gives general conditions under which a function in $H^{2}$ acts isometrically as a multiplication on $\mathcal{H}(\phi)$ when $\phi$ is an inner function.

For $w \in D$, let $\alpha_{w}=(w-z)(1-\bar{w} z)^{-1}$ and $g_{w}=\left(1-|w|^{2}\right)^{1 / 2}(1-$ $\bar{w} z)^{-1}$. Thus $\alpha_{w}$ is a disk automorphism, and $g_{w}$ is just $k_{w}$ normalized in $H^{2}$, that is, $g_{w}=k_{w} /\left\|k_{w}\right\|$.

Lemma 7. Let $b$ be a function in the closed unit ball in $H^{\infty}$. Then, for any $w \in D$,

$$
T_{g_{w} \circ b}\left(1-T_{b} T_{\bar{b}}\right) T_{\bar{g}_{w} \circ b}=1-T_{\alpha_{w} \circ b} T_{\bar{\alpha}_{w} \circ b} .
$$


Proof. Fix $w \in D$. For convenience, let $h=(1-\bar{w} b)^{-1}$. Thus, $g_{w} \circ b=\left(1-|w|^{2}\right)^{1 / 2} h$ and $\alpha_{w} \circ b=(w-b) h$. The proof is just a calculation:

$$
\begin{aligned}
T_{g_{w} \circ b}(1 & \left.-T_{b} T_{\bar{b}}\right) T_{\bar{g}_{w} \circ b} \\
& =\left(1-|w|^{2}\right)\left(T_{h} T_{\bar{h}}-T_{b h} T_{\bar{b} \bar{h}}\right) \\
& =\left(T_{h}-\bar{w} T_{b h}\right)\left(T_{\bar{h}}-w T_{\bar{b} \bar{h}}\right)-\left(w T_{h}-T_{b h}\right)\left(\bar{w} T_{\bar{h}}-T_{\bar{b} \bar{h}}\right) \\
& =T_{(1-\bar{w} b) h} T_{(1-w \bar{b}) \bar{h}}-T_{(w-b) h} T_{(\bar{w}-\bar{b}) \bar{h}} \\
& =1-T_{\alpha_{w} \circ b} T_{\bar{\alpha}_{w} \circ b} .
\end{aligned}
$$

LEMMA 8. Let $b_{1}$ and $b_{2}$ be functions in the closed unit ball in $H^{\infty}$, both vanishing at the origin. If there exists an isometric multiplier $m$ from $\mathcal{H}\left(b_{1}\right)$ onto $\mathcal{H}\left(b_{2}\right)$, then $b_{2}=\lambda b_{1}$ for some constant $\lambda \in T$, and $m$ is a unimodular constant.

Proof. Suppose $m$ is an isometric multiplier from $\mathcal{H}\left(b_{1}\right)$ onto $\mathcal{H}\left(b_{2}\right)$. Since $1=k_{0}^{b_{1}} \in \mathcal{H}\left(b_{1}\right)$ and $1=k_{0}^{b_{2}} \in \mathcal{H}\left(b_{2}\right)$, it follows that $m \in \mathcal{H}\left(b_{2}\right)$ and $m^{-1} \in \mathcal{H}\left(b_{1}\right)$. Since multiplication by $m$ is an isometry on $\mathcal{H}\left(b_{1}\right),\langle m, 1\rangle_{b_{2}}=\left\langle 1, m^{-1}\right\rangle_{b_{1}}$, that is, $m(0)=\overline{m^{-1}(0)}$. Hence $|m(0)|=1$. Furthermore, $\|m\| \leq\|m\|_{b_{2}}=\|1\|_{b_{1}}=1$. Thus $\|m\| \leq|m(0)|$, which implies $m=m(0)$, a unimodular constant. It follows that $\mathcal{H}\left(b_{2}\right)=\mathcal{H}\left(b_{1}\right)$. Equivalently, $b_{2}=\lambda b_{1}$ for some constant $\lambda \in T$.

The following lemma is from a general theorem by R. G. Douglas [2]. The symbol $\perp$ is used in the usual way for the relation of orthogonality, and the null space of a linear operator $A$ is denoted by $\mathcal{N} A$.

LEMMA 9. Let $A$ and $B$ be bounded linear operators on a Hilbert space $\mathcal{K}$. If $A A^{*} \leq B B^{*}$, then:

(i) $A \mathcal{K} \subseteq B \mathcal{K}$

(ii) There exists a contraction $C$ on $\mathcal{K}$ such that $A=B C$ and $C$ maps $(\mathcal{N} A)^{\perp}$ into $(\mathcal{N} B)^{\perp}$.

THEOREM 10. (a) Let $b$ be a function in the closed unit ball in $H^{\infty}$, and let $w \in D$. Define $\alpha_{w}$ and $g_{w}$ as above. Then $g_{w} \circ b$ is an 
isometric multiplier from $\mathcal{H}(b)$ onto $\mathcal{H}\left(\alpha_{w} \circ b\right)$.

(b) Let $b_{1}$ and $b_{2}$ be functions in the closed unit ball in $H^{\infty}$, neither of which is a unimodular constant. If there exists an isometric multiplier from $\mathcal{H}\left(b_{1}\right)$ onto $\mathcal{H}\left(b_{2}\right)$, then $b_{2}=\tau \circ b_{1}$ for some disk automorphism $\tau$. If $m_{1}$ and $m_{2}$ are two such multipliers, then $m_{2}=\gamma m_{1}$ for some constant $\gamma$ of unit modulus.

Proof. (a) Let $A=T_{g_{w} \circ b}\left(1-T_{b} T_{\bar{b}}\right)^{1 / 2}$ and $B=\left(1-T_{\alpha_{w} \circ b} T_{\bar{\alpha}_{w} \circ b}\right)^{1 / 2}$. Then, according to Lemma $7, A A^{*}=B B^{*}$. Hence, by Lemma 9 , $A H^{2}=B H^{2}$, that is, $T_{g_{w} \circ b} \mathcal{H}(b)=\mathcal{H}\left(\alpha_{w} \circ b\right)$. Thus $g_{w} \circ b$ is a multiplier from $\mathcal{H}(b)$ onto $\mathcal{H}\left(\alpha_{w} \circ b\right)$.

Now it will be shown that $T_{g_{w} \text { ob }}$ acts isometrically on $\mathcal{H}(b)$. By Lemma 9, there exists a contraction $C$ on $H^{2}$ such that $A=B C$ and $C$ maps $(\mathcal{N} A)^{\perp}$ into $(\mathcal{N} B)^{\perp}$. Let $f \in \mathcal{H}(b)$, and let $h$ be the unique function in $H^{2}$ such that $\left(1-T_{b} T_{\bar{b}}\right)^{1 / 2} h=f$ and $h \perp \mathcal{N}\left(1-T_{b} T_{\bar{b}}\right)^{1 / 2}$. Since $T_{g_{w} \text { ob }}$ is injective, $\mathcal{N} A=\mathcal{N}\left(1-T_{b} T_{\bar{b}}\right)^{1 / 2}$. Thus $h \perp \mathcal{N} A$, and hence $C h \perp \mathcal{N} B$. Also, $B C h=A h=T_{g_{w} \circ b} f$. By definition of the norms on $\mathcal{H}(b)$ and $\mathcal{H}\left(\alpha_{w} \circ b\right),\|f\|_{b}=\|h\|$ and $\left\|T_{g_{w} \circ b} f\right\|_{\alpha_{w} \circ b}=$ $\|C h\|$. Since $C$ is a contraction, $\left\|T_{g_{w} \circ b} f\right\|_{\alpha_{w} \text { ob }} \leq\|f\|_{b}$. By a similar argument, if $f \in \mathcal{H}\left(\alpha_{w} \circ b\right)$, then $\left\|T_{\left(g_{w} \circ b\right)^{-1}} f\right\|_{b} \leq\|f\|_{\alpha_{w} \circ b}$. It follows that the norms in these inequalities are in fact equal.

(b) Let $w_{1}=b_{1}(0)$ and $w_{2}=b_{2}(0)$. Using part (a), $g_{w_{1}} \circ b_{1}$ is an isometric multiplier from $\mathcal{H}\left(b_{1}\right)$ onto $\mathcal{H}\left(\alpha_{w_{1}} \circ b_{1}\right)$, and $g_{w_{2}} \circ b_{2}$ is an isometric multiplier from $\mathcal{H}\left(b_{2}\right)$ onto $\mathcal{H}\left(\alpha_{w_{2}} \circ b_{2}\right)$. If $m$ is an isometric multiplier from $\mathcal{H}\left(b_{1}\right)$ onto $\mathcal{H}\left(b_{2}\right)$, then $m\left(g_{w_{1}} \circ b_{1}\right)^{-1}\left(g_{w_{2}} \circ b_{2}\right)$ is an isometric multiplier from $\mathcal{H}\left(\alpha_{w_{1}} \circ b_{1}\right)$ onto $\mathcal{H}\left(\alpha_{w_{2}} \circ b_{2}\right)$. Since both $\alpha_{w_{1}} \circ b_{1}$ and $\alpha_{w_{2}} \circ b_{2}$ vanish at the origin, Lemma 8 applies: $\alpha_{w_{2}} \circ b_{2}=$ $\lambda \alpha_{w_{1}} \circ b_{1}$, for some $\lambda \in T$, and the multiplier $m\left(g_{w_{1}} \circ b_{1}\right)^{-1}\left(g_{w_{2}} \circ b_{2}\right)$ is a unimodular constant. Now $b_{2}=\tau \circ b_{1}$, where $\tau=\alpha_{w_{2}} \circ\left(\lambda \alpha_{w_{1}}\right)$.

If $m_{1}$ and $m_{2}$ are two isometric multipliers from $\mathcal{H}\left(b_{1}\right)$ onto $\mathcal{H}\left(b_{2}\right)$, then both $m_{1}\left(g_{w_{1}} \circ b_{1}\right)^{-1}\left(g_{w_{2}} \circ b_{2}\right)$ and $m_{2}\left(g_{w_{1}} \circ b_{1}\right)^{-1}\left(g_{w_{2}} \circ b_{2}\right)$ are unimodular constants, and hence so is $m_{2} / m_{1}$.

4. Uniqueness. The Hilbert spaces $\mathcal{H}(b)$ provide a natural setting for isometric multipliers, but not for multipliers in general. Useful results about non-isometric multipliers emerge only for the special case of spaces $\mathcal{H}(\phi)$ corresponding to inner functions $\phi$, that is, the $S^{*}$-invariant subspaces of $H^{2}$. It will be shown that if a mul- 
tiplier exists between given subspaces $\mathcal{H}\left(\phi_{1}\right)$ and $\mathcal{H}\left(\phi_{2}\right)$, it is unique up to multiplication by a constant (Corollary 13). For spaces $\mathcal{H}(b)$ in general, multipliers are far from unique. For example, if $b$ is any function in the open unit ball in $H^{\infty}$, then the set $\mathcal{H}(b)$ is equal to $H^{2}$, and any invertible function in $H^{\infty}$ is a multiplier from $\mathcal{H}(b)$ onto itself.

LEMMA 11. Let $\phi$ be an inner function, and let $m \in H^{2}$. Then

$$
\left|\left\langle m S^{*} \phi, \phi S^{*} m\right\rangle\right| \geq(1-|\phi(0)|)\left\|S^{*} m\right\|^{2} \text {. }
$$

Proof. The algebraic identity $m S^{*} \phi=\phi S^{*} m-\phi(0) S^{*} m+m(0) S^{*} \phi$ can be used to show that

$$
\left\langle m S^{*} \phi, \phi S^{*} m\right\rangle=\left\|S^{*} m\right\|^{2}-\phi(0)\left\langle S^{*} m, \phi S^{*} m\right\rangle .
$$

Using the Cauchy-Schwarz inequality, $\left|\left\langle S^{*} m, \phi S^{*} m\right\rangle\right| \leq\left\|S^{*} m\right\|$. $\left\|\phi S^{*} m\right\|=\left\|S^{*} m\right\|^{2}$, and hence

$$
\left|\left\langle m S^{*} \phi, \phi S^{*} m\right\rangle\right| \geq(1-|\phi(0)|)\left\|S^{*} m\right\|^{2} .
$$

Proposition 12. Let $\phi$ be a nonconstant inner function, and let $m \in H^{2}, m \neq 0$. The following statements are equivalent:

(i) $m \mathcal{H}(\phi)=\mathcal{H}(\phi)$,

(ii) $m \mathcal{H}(\phi) \subseteq \mathcal{H}(\phi)$,

(iii) $m S^{*} \phi \in \mathcal{H}(\phi)$,

(iv) $m$ is a constant function.

Proof. Clearly (iv) $\Longrightarrow\left(\right.$ i) $\Longrightarrow$ (ii). Also, (ii) $\Longrightarrow\left(\right.$ iii), since $S^{*} \phi \in$ $\mathcal{H}(\phi)$. If (iii), then $\left\langle m S^{*} \phi, \phi S^{*} m\right\rangle=0$, from which, using Lemma 11, $(1-|\phi(0)|)\left\|S^{*} m\right\|^{2}=0$. Since $\phi$ is not constant, $1-|\phi(0)|>0$. Therefore $S^{*} m=0$, which implies (iv).

COROLlaRY 13. Let $\phi$ and $\psi$ be nonconstant inner functions. If there exists a multiplier $m$ from $\mathcal{H}(\phi)$ onto $\mathcal{H}(\psi)$, then the set of all such multipliers is $\{\lambda m: \lambda \in C, \lambda \neq 0\}$. 
Proof. Suppose there exists a multiplier from $\mathcal{H}(\phi)$ onto $\mathcal{H}(\psi)$. Choose one such multiplier $m$. If $\lambda \in C$ and $\lambda \neq 0$, then clearly $\lambda m$ is also such a multiplier. On the other hand, if $\tilde{m}$ is any multiplier from $\mathcal{H}(\phi)$ onto $\mathcal{H}(\psi)$, then $\tilde{m} \mathcal{H}(\phi)=\mathcal{H}(\psi)=m \mathcal{H}(\phi)$, and hence $\tilde{m} m^{-1} \mathcal{H}(\phi)=\mathcal{H}(\phi)$. By Corollary $4, \tilde{m} m^{-1} \in H^{2}$. Then, by Proposition $12, \tilde{m} m^{-1}$ is a constant, which is obviously nonzero.

5. Existence. For which inner functions $\phi$ and $\psi$ does there exist a multiplier between $\mathcal{H}(\phi)$ and $\mathcal{H}(\psi)$ ? In case such a multiplier exists, how is it related to $\phi$ and $\psi$ ?

The next theorem provides a necessary condition for the existence of a multiplier. In the proof of the theorem the following notation will be used: if $\phi$ is an inner function, $S_{\phi}^{*}$ will denote the restriction of $S^{*}$ to the invariant subspace $\mathcal{H}(\phi)$, and $\operatorname{spec}_{e} S_{\phi}^{*}$ will denote the essential spectrum of $S_{\phi}^{*}$. According to [6] (pp. 62-65), $\overline{\operatorname{spec}_{e} S_{\phi}^{*}}$ is the set of singularities of $\phi$ on $T$.

THEOREM 14. Let $\phi$ and $\psi$ be inner functions. If there exists a multiplier between $\mathcal{H}(\phi)$ and $\mathcal{H}(\psi)$, then $\phi$ and $\psi$ have the same set of singularities on the unit circle.

Proof. Let $m$ be a multiplier from $\mathcal{H}(\phi)$ onto $\mathcal{H}(\psi)$. Let $A$ denote the restriction of $T_{m}$ to $\mathcal{H}(\phi)$. By Proposition $5, A$ is bounded. Restricting the domains of the operators in the commutation relation $S^{*} T_{m}-T_{m} S^{*}=\left(S^{*} m\right) \otimes 1$ gives $S_{\psi}^{*} A-A S_{\phi}^{*}=\left(S^{*} m\right) \otimes 1$. (Each side of this equation is regarded as an operator on $\mathcal{H}(\phi)$.) Hence $A^{-1} S_{\psi}^{*} A-S_{\phi}^{*}=m^{-1}\left(S^{*} m\right) \otimes 1$. Since the operator on the right side of this equation has rank one, and is therefore compact, the operators $A^{-1} S_{\psi}^{*} A$ and $S_{\phi}^{*}$ on the left side belong to the same coset in the Calkin algebra $\mathcal{L}(\mathcal{H}(\phi)) / \mathcal{K}(\mathcal{H}(\phi))$, and therefore have the same essential spectrum. Furthermore, $A^{-1} S_{\psi}^{*} A$ and $S_{\psi}^{*}$ have the same essential spectrum because they are similar. Thus $\operatorname{spec}_{e} S_{\phi}^{*}=\operatorname{spec}_{e} S_{\psi}^{*}$. As noted above, this implies that $\phi$ and $\psi$ have the same set of singularities on $T$.

Approaching the existence question from a different perspective, let us regard $m$ as given and search for inner functions $\phi$ and $\psi$ to 
satisfy $m \mathcal{H}(\phi)=\mathcal{H}(\psi)$. A clue is provided by the algebraic relationships involved: if $m$ is an analytic function on $D$ with no zeros, multiplication by $m^{-1}$ maps the linear span of $\left\{S^{* n} m\right\}_{n=1}^{\infty}$ onto the linear span of $\left\{S^{* n}\left(m^{-1}\right)\right\}_{n=1}^{\infty}$. This is easily seen by induction once it is noted that for any $n \in N$,

$$
m^{-1} S^{*(n+1)} m=S^{*}\left(m^{-1} S^{* n} m\right)-\left(S^{* n} m\right)(0) \cdot S^{*}\left(m^{-1}\right) .
$$

Thus we are led to investigate the invariant subspaces generated by $S^{*} m$ and $S^{*}\left(m^{-1}\right)$.

For $f \in H^{2}$, the $S^{*}$-invariant subspace generated by $f$ is the closed linear span of $\left\{S^{* n} f\right\}_{n=0}^{\infty}$. If this subspace is the entire space $H^{2}, f$ is called cyclic, or more precisely, $S^{*}$-cyclic. If $f$ is noncyclic, the $S^{*}$ invariant subspace generated by $f$ is $\mathcal{H}(\phi)$ for some inner function $\phi$, determined by $f$ uniquely up to a constant. The same statement applies with $f$ replaced by $S^{*} f$ (which is obviously noncyclic whenever $f$ is noncyclic). Define $\Theta(f)$ to be the inner function (determined up to a constant) such that $\mathcal{H}(\Theta(f))$ is the $S^{*}$-invariant subspace generated by $S^{*} f$. Note that for any inner function $\phi, \Theta(\phi)=\phi$, since the $S^{*}$-invariant subspace generated by $S^{*} \phi$ is $\mathcal{H}(\phi)$.

The following lemmas are not new: Lemma 15 is essentially Theorem 3.1 .5 in [7] (cf. [6] pp. 30,31) and Lemma 16 is similar to Theorem 3.1.1 in [7]. Proofs are given here because the lemmas are crucial for Theorem 17.

LEMMA 15. Let $\phi$ be an inner function. Then, for any $f \in H^{2}$, $S^{*} f \in \mathcal{H}(\phi)$ if and only if $\phi \bar{f} \in H^{2}$.

Proof. First note that $\mathcal{H}(\phi)$ is the null space of the Toeplitz operator $T_{\bar{\phi}}$. This follows from the fact that $T_{\bar{\phi}}$ is the adjoint of $T_{\phi}$, which has range $\phi H^{2}$.

Let $f \in H^{2}$. Then $S^{*} f \in \mathcal{H}(\phi)$ if and only if $T_{\bar{\phi}} S^{*} f=0$. Since $T_{\bar{\phi}} S^{*} f=T_{\bar{z} \bar{\phi}} f=P^{+}(\bar{z} \bar{\phi} f)$, where $P^{+}$denotes the orthogonal projection of $L^{2}$ onto $H^{2}, T_{\bar{\phi}} S^{*} f=0$ if and only if $\bar{z} \bar{\phi} f \in \overline{z H^{2}}$, that is, $\phi \bar{f} \in H^{2}$.

LEMMA 16. Let $f$ be an outer function in $H^{2}$ such that $f^{-1} \in$ $H^{2}$. Then $f$ is cyclic if and only if $f^{-1}$ is cyclic. For any inner 
functions $\phi$ and $\psi$, the following statements are equivalent:

(i) $f$ is noncyclic, and there exists an inner function $\xi$ such that $\phi=\xi \Theta\left(f^{-1}\right)$ and $\psi=\xi \Theta(f)$ (up to constants).

(ii) $(\bar{f} \psi) /(f \phi)$ is constant on $T$.

If $f$ is noncyclic, then $[\bar{f} \Theta(f)] /\left[f \Theta\left(f^{-1}\right)\right]$ is constant on $T$, and $\Theta(f)$ and $\Theta\left(f^{-1}\right)$ are relatively prime.

Proof. Suppose $f$ is noncyclic. Then $\Theta(f)$ is defined, and $S^{*} f \in$ $\mathcal{H}(\Theta(f))$. By Lemma 15, the function $\Theta(f) \cdot \bar{f}$ is in $H^{2}$. Its outer factor has modulus $|\Theta(f) \cdot \bar{f}|=|f|$ and therefore equals $f$. So $\Theta(f) \cdot \bar{f}=\varrho f$ for some inner function $\varrho$. Rearranging this equation, $\varrho \cdot \bar{f}^{-1}=\Theta(f) \cdot f^{-1}$. Thus $\varrho \cdot \bar{f}^{-1} \in H^{2}$, which means $S^{*}\left(f^{-1}\right) \in$ $\mathcal{H}(\varrho)$. This implies that $f^{-1}$ is not cyclic and also that $\mathcal{H}\left(\Theta\left(f^{-1}\right)\right)$ is contained in $\mathcal{H}(\varrho)$. Hence $\Theta\left(f^{-1}\right)$ divides $\varrho$. By the same argument with $f^{-1}$ in place of $f, \Theta\left(f^{-1}\right) \cdot \bar{f}^{-1}=\sigma f^{-1}$ for some inner function $\sigma$ divisible by $\Theta(f)$. So $\Theta\left(f^{-1}\right) \cdot \Theta(f)=\varrho \sigma$. Since $\Theta\left(f^{-1}\right)$ divides $\varrho$ and $\Theta(f)$ divides $\sigma$, it follows that $\varrho=\Theta\left(f^{-1}\right)$ and $\sigma=\Theta(f)$ (up to constants). Then $[\bar{f} \Theta(f)] /\left[f \Theta\left(f^{-1}\right)\right]$ is constant.

In the course of the above argument it was shown that $f^{-1}$ is noncyclic whenever $f$ is noncyclic. It follows that $f$ is cyclic if and only if $f^{-1}$ is cyclic.

Let $\phi$ and $\psi$ be inner functions. Suppose $(\bar{f} \psi) /(f \phi)$ is constant on $T$. Then $\psi \bar{f} \in H^{2}$, and therefore $S^{*} f \in \mathcal{H}(\psi)$ (Lemma 15). So $f$ is noncyclic, and $\mathcal{H}(\Theta(f)) \subseteq \mathcal{H}(\psi)$, that is, $\Theta(f)$ divides $\psi$. Similarly, $\Theta\left(f^{-1}\right)$ divides $\phi$. Let $\xi=\phi / \Theta\left(f^{-1}\right)$. Then

$$
\frac{\psi}{\xi \Theta(f)}=\frac{\bar{f} \psi}{f \phi} \cdot \frac{f \Theta\left(f^{-1}\right)}{\bar{f} \Theta(f)} .
$$

The right side of this equation is a constant. Thus, up to a constant, $\psi=\xi \Theta(f)$. This proves (ii) $\Longrightarrow(\mathrm{i})$. Conversely, (i) implies $(\bar{f} \psi) /(f \phi)=[\bar{f} \Theta(f)] /\left[f \Theta\left(f^{-1}\right)\right]$, which has been shown to be constant on $T$.

Now suppose $\eta$ is a common divisor of $\Theta\left(f^{-1}\right)$ and $\Theta(f)$, and let $\phi=\Theta\left(f^{-1}\right) / \eta$ and $\psi=\Theta(f) / \eta$. Then

$$
(\bar{f} \psi) /(f \phi)=[\bar{f} \Theta(f)] /\left[f \Theta\left(f^{-1}\right)\right],
$$

which is constant. Therefore $\Theta\left(f^{-1}\right)$ divides $\phi$ and $\Theta(f)$ divides $\psi$, which means that $\eta$ must be constant. Thus $\Theta(f)$ and $\Theta\left(f^{-1}\right)$ are relatively prime. 
Now these ideas will be applied to the multiplier problem.

THEOREM 17. Let $\phi$ and $\psi$ be nonconstant inner functions. (a) If $m$ is a multiplier from $\mathcal{H}(\phi)$ onto $\mathcal{H}(\psi)$, then $(\psi \bar{m}) /(\phi m)$ is constant on $T, m$ is noncyclic, and there is some inner function $\xi$ such that $\phi=\xi \Theta\left(m^{-1}\right)$ and $\psi=\xi \Theta(m)$.

(b) If $m$ is an analytic function on $D$ such that $m \mathcal{H}(\phi) \subseteq H^{2}$ and $m^{-1} \mathcal{H}(\psi) \subseteq H^{2}$, and if $(\psi \bar{m}) /(\phi m)$ is constant on $T$, then $m$ is a multiplier from $\mathcal{H}(\phi)$ onto $\mathcal{H}(\psi)$.

Proof. (a) Suppose $m \mathcal{H}(\phi)=\mathcal{H}(\psi)$. A calculation is needed:

$$
\begin{aligned}
T_{\bar{m} \bar{\phi} \psi} S-S T_{\bar{m} \bar{\phi} \psi} & =\left(T_{\bar{m} \bar{\phi}} S-S T_{\bar{m} \bar{\phi}}\right) T_{\psi} \\
& =\left(1 \otimes\left[S^{*}(m \phi)\right]\right) T_{\psi} \\
& =1 \otimes\left[T_{\bar{\psi}} S^{*}(m \phi)\right] .
\end{aligned}
$$

Now note that $S^{*}(m \phi) \in \mathcal{H}(\psi)$, since $S^{*}(m \phi)=m S^{*} \phi+\phi(0) S^{*} m$, and hence $T_{\bar{\psi}} S^{*}(m \phi)=0$. So $T_{\bar{m} \bar{\phi} \psi} S-S T_{\bar{m} \bar{\phi} \psi}=0$, that is, $T_{\bar{m} \bar{\phi} \psi}$ commutes with $S$. This implies $\bar{m} \phi \psi \in H^{2}$. Since $m \mathcal{H}(\phi) \subseteq H^{2}$, it follows that $\bar{m} \bar{\phi} \psi \mathcal{H}(\phi) \subseteq H^{2}$. In fact $\bar{m} \bar{\phi} \psi \mathcal{H}(\phi) \subseteq \mathcal{H}(\bar{\psi})$, as shown by the following calculation: for all $f \in \mathcal{H}(\phi)$ and $h \in H^{\infty}$, $\langle\bar{m} \bar{\phi} \psi f, \psi h\rangle=\langle f, \phi m h\rangle=0$. The same argument can be applied to $m^{-1}$ as a multiplier from $\mathcal{H}(\psi)$ onto $\mathcal{H}(\phi)$, resulting in the inclusion $\bar{m}^{-1} \bar{\psi} \phi \mathcal{H}(\psi) \subseteq \mathcal{H}(\phi)$. Thus $\bar{m} \bar{\phi} \psi \mathcal{H}(\phi)=\mathcal{H}(\psi)$. Since multipliers are unique up to constants (Corollary 13), $\bar{m} \bar{\phi} \psi / m$ is constant, that is, $(\psi \bar{m}) /(\phi m)$ is constant. Then, by Lemma $16, m$ is noncyclic, and there is some inner function $\xi$ such that $\phi=\xi \Theta\left(m^{-1}\right)$ and $\psi=\xi \Theta(m)$.

(b) Let $m$ be an analytic function on $D$ such that $m \mathcal{H}(\phi) \subseteq H^{2}$ and $m^{-1} \mathcal{H}(\psi) \subseteq H^{2}$. Since $\mathcal{H}(\phi)$ contains functions which are invertible in $H^{\infty}$ (for example, $1-\overline{\phi(0)} \phi$ ), the inclusion $m \mathcal{H}(\phi) \subseteq H^{2}$ implies $m \in H^{2}$. Similarly, $m^{-1} \in H^{2}$, and therefore $m$ is outer.

Suppose $(\psi \bar{m}) /(\phi m)=\lambda$, where $\lambda$ is a constant. Let $f \in \mathcal{H}(\phi)$. If $h \in H^{\infty}$, then $\langle m f, \psi h\rangle=\langle f, \psi \bar{m} h\rangle=\langle f, \lambda \phi m h\rangle=0$. Thus $m f \perp$ $\psi H^{\infty}$, and therefore $m f \in \mathcal{H}(\psi)$. This proves $m \mathcal{H}(\phi) \subseteq \mathcal{H}(\psi)$. Since $\left(\phi \bar{m}^{-1}\right) /\left(\psi m^{-1}\right)$ is a constant (namely $\left.\bar{\lambda}\right)$, the same argument can be applied with $\phi$ and $\psi$ interchanged and $m^{-1}$ in place of $m$, proving that $m^{-1} \mathcal{H}(\psi) \subseteq \mathcal{H}(\phi)$. Thus $m \mathcal{H}(\phi)=\mathcal{H}(\psi)$. 
CoRollary 18. Let $m \in H^{\infty}$ such that $m^{-1} \in H^{\infty}$. If $m$ is noncyclic, then $m$ is a multiplier from $\mathcal{H}\left(\Theta\left(m^{-1}\right)\right)$ onto $\mathcal{H}(\Theta(m))$. For any nonconstant inner functions $\phi$ and $\psi$, the following statements are equivalent:

(i) $m$ is a multiplier from $\mathcal{H}(\phi)$ onto $\mathcal{H}(\psi)$,

(ii) $(\psi \bar{m}) /(\phi m)$ is constant on $T$,

(iii) $m$ is noncyclic, and there is some inner function $\xi$ such that $\phi=\xi \Theta\left(m^{-1}\right)$ and $\psi=\xi \Theta(m)$.

Proof. If $m$ is noncyclic, then by Lemma $16,[\bar{m} \Theta(m)] /\left[m \Theta\left(m^{-1}\right)\right]$ is constant. Clearly $m \mathcal{H}\left(\Theta\left(m^{-1}\right)\right) \subseteq H^{2}$ (since $m \in H^{\infty}$ ) and $m^{-1} \mathcal{H}(\Theta(m)) \subseteq H^{2}$ (since $\left.m^{-1} \in H^{\infty}\right)$. Hence, by Theorem $17(\mathrm{~b})$, $m$ is a multiplier from $\mathcal{H}\left(\Theta\left(m^{-1}\right)\right)$ onto $\mathcal{H}(\Theta(m))$. If $\phi$ and $\psi$ are nonconstant inner functions, then (i) $\Leftrightarrow$ (ii) by Theorem 17 , and (ii) $\Leftrightarrow$ (iii) by Lemma 16 .

COROllaRY 19. Let $m$ be an analytic function on $D$, and let $\phi, \psi$ and $\xi$ be nonconstant inner functions. Then

$$
m \mathcal{H}(\phi \xi)=\mathcal{H}(\psi \xi) \Longrightarrow m \mathcal{H}(\phi)=\mathcal{H}(\psi) \text {. }
$$

If both $m$ and $m^{-1}$ are in $H^{\infty}$, then

$$
m \mathcal{H}(\phi \xi)=\mathcal{H}(\psi \xi) \Longleftrightarrow m \mathcal{H}(\phi)=\mathcal{H}(\psi) .
$$

Proof. Suppose $m \mathcal{H}(\phi \xi)=\mathcal{H}(\psi \xi)$. By Theorem 17(a), $(\psi \xi \bar{m}) /(\phi \xi m)=\lambda$ for some constant $\lambda$. Then $(\psi \bar{m}) /(\phi m)=\lambda$. Furthermore, $m \mathcal{H}(\phi) \subseteq m \mathcal{H}(\phi \xi)=\mathcal{H}(\psi \xi)$, and therefore $m \mathcal{H}(\phi) \subseteq H^{2}$. Similarly, $m^{-1} \mathcal{H}(\psi) \subseteq H^{2}$. By Theorem $17(\mathrm{~b}), m \mathcal{H}(\phi)=\mathcal{H}(\psi)$.

If $m$ and $m^{-1}$ are bounded, the equivalence $m \mathcal{H}(\phi \xi)=\mathcal{H}(\psi \xi) \Longleftrightarrow$ $m \mathcal{H}(\phi)=\mathcal{H}(\psi)$ follows immediately from Corollary 18 .

COROLLARY 20. Let $\phi$ and $\psi$ be nonconstant inner functions which are relatively prime. If $m$ is a multiplier from $\mathcal{H}(\phi)$ onto $\mathcal{H}(\psi)$, then $\phi=\Theta\left(m^{-1}\right)$ and $\psi=\Theta(m)$ (up to constants).

Proof. Suppose $m \mathcal{H}(\phi)=\mathcal{H}(\psi)$. By Theorem 17(a), there is some inner function $\xi$ such that $\phi=\xi \Theta\left(m^{-1}\right)$ and $\psi=\xi \Theta(m)$. Since $\phi$ and $\psi$ are relatively prime, $\xi=1$. 
COROLlaRY 21. Let $\phi$ and $\psi$ be inner functions one of which is a nonconstant multiple of the other. Then there is no multiplier between $\mathcal{H}(\phi)$ and $\mathcal{H}(\psi)$.

Proof. Suppose $\phi=\xi \psi$ for some inner function $\xi$. The aim is to show that if there is a multiplier between $\mathcal{H}(\phi)$ and $\mathcal{H}(\psi)$, then $\xi$ must be constant. It is tempting to appeal to Corollary 19 to reduce the problem to the trivial case of a multiplier between $\mathcal{H}(\xi)$ and $\mathcal{H}(1)=\{0\}$, but this is not legitimate because the inner functions in Corollary 19 are supposed to be nonconstant.

Let $m$ be a multiplier from $\mathcal{H}(\phi)$ onto $\mathcal{H}(\psi)$. By the argument at the beginning of the proof of Theorem 17, the operator $T_{\bar{m} \bar{\phi} \psi}$ commutes with $S$. Note that $T_{\bar{m} \bar{\phi} \psi}=T_{\bar{m} \bar{\xi}}$, and so $T_{\bar{m} \bar{\xi}} S-S T_{\bar{m} \bar{\xi}}=0$. Since $T_{\bar{m} \bar{\xi}} S-S T_{\bar{m} \bar{\xi}}=1 \otimes\left[S^{*}(m \xi)\right]$, it follows that $S^{*}(m \xi)=0$, which means that $m \xi$ is constant. This is possible only if both $m$ and $\xi$ are constant.

6. Evaluation kernels as multipliers. For $w \in D$, let $\beta_{w}=$ $\lambda_{w}(z-w)(1-\bar{w} z)^{-1}$, where $\lambda_{w}$ is a normalization constant which, for purposes of this section, can be chosen arbitrarily. The subspace $\mathcal{H}\left(\beta_{w}\right)$ is one-dimensional, spanned by $k_{w}$. (Recall that $k_{w}$ denotes the kernel in $H^{2}$ for evaluation at $w$.)

Proposition 22. Let $w$ and $w^{\prime}$ be any two points in $D$. Then, for any inner function $\xi,\left(k_{w^{\prime}} / k_{w}\right) \mathcal{H}\left(\beta_{w} \xi\right)=\mathcal{H}\left(\beta_{w^{\prime}} \xi\right)$.

Proof. Since $\mathcal{H}\left(\beta_{w}\right)$ is spanned by $k_{w}$ and $\mathcal{H}\left(\beta_{w^{\prime}}\right)$ is spanned by $k_{w^{\prime}}$, it is clear that $\left(k_{w^{\prime}} / k_{w}\right) \mathcal{H}\left(\beta_{w}\right)=\mathcal{H}\left(\beta_{w^{\prime}}\right)$. Then, by Corollary 19, $\left(k_{w^{\prime}} / k_{w}\right) \mathcal{H}\left(\beta_{w} \xi\right)=\mathcal{H}\left(\beta_{w^{\prime}} \xi\right)$ for any inner function $\xi$.

Corollary 23. Let $n \in N$, and let $\left(w_{j}\right)_{j=1}^{n}$ and $\left(w_{j}^{\prime}\right)_{j=1}^{n}$ be any two sequences of length $n$ in $D$. Let $\phi=\prod_{j=1}^{n} \beta_{w_{j}}$, let $\psi=\prod_{j=1}^{n} \beta_{w_{j}^{\prime}}$, and let $m=\prod_{j=1}^{n}\left(k_{w_{j}^{\prime}} / k_{w_{j}}\right)$. Then $m \mathcal{H}(\phi)=\mathcal{H}(\psi)$.

Proof. Apply Proposition 22 repeatedly.

Corollary 24. Let $\phi$ and $\psi$ be finite Blaschke products. The 
following statements are equivalent:

(i) There exists a multiplier between $\mathcal{H}(\phi)$ and $\mathcal{H}(\psi)$,

(ii) $\mathcal{H}(\phi)$ and $\mathcal{H}(\psi)$ have the same dimension,

(iii) $\phi$ and $\psi$ have the same number of zeros (counting multiplicities).

Proof. If $\left\{f_{1}, f_{2}, \ldots, f_{n}\right\}$ is a linearly independent set of analytic functions and $g$ is an analytic function which is not the zero function, then $\left\{g f_{1}, g f_{2}, \ldots, g f_{n}\right\}$ is linearly independent. From this it follows that (i) $\Rightarrow$ (ii). The equivalence (ii) $\Leftrightarrow$ (iii) is a fact about $S^{*}$ invariant subspaces, noted already in Section 1 . If (iii) holds, then Corollary 23 gives a multiplier between $\mathcal{H}(\phi)$ and $\mathcal{H}(\psi)$.

The following proposition shows how to use the kernel $k_{w}^{\phi}$ as a multiplier even if $\phi$ does not have a zero at $w$.

Proposition 25. Let $\phi$ be an inner function and $w \in D$. Then $\left(k_{w}^{\phi}\right)^{-1} \mathcal{H}(\phi)=\mathcal{H}(\psi)$, where $\psi=z \beta_{w}^{-1}[\phi-\phi(w)][1-\overline{\phi(w)} \phi]^{-1}$.

Proof. Let $\xi=[\phi-\phi(w)] \cdot[1-\overline{\phi(w)} \phi]^{-1}$. Note that $\xi(w)=0$, and so $\beta_{w}$ divides $\xi$. Let $\psi=z \xi / \beta_{w}$. By Theorem 10(a), the function $\left(1-|\phi(w)|^{2}\right)^{1 / 2} \cdot[1-\overline{\phi(w)} \phi]^{-1}$ is an isometric multiplier from $\mathcal{H}(\phi)$ onto $\mathcal{H}(\xi)$. Hence $[1-\overline{\phi(w)} \phi]^{-1} \mathcal{H}(\phi)=\mathcal{H}(\xi)$. Applying Proposition $22, k_{w}^{-1} \mathcal{H}(\xi)=\mathcal{H}(\psi)$. Since $k_{w}^{\phi}=[1-\overline{\phi(w)} \phi] k_{w}$, it follows that $\left(k_{w}^{\phi}\right)^{-1} \mathcal{H}(\phi)=\mathcal{H}(\psi)$.

\section{Multipliers via infinite products.}

LEMMA 26. Let $\left(w_{k}\right)_{k=1}^{\infty}$ and $\left(w_{k}^{\prime}\right)_{k=1}^{\infty}$ be any two sequences in $D$. If any of the following series converges, then they all converge.
(i) $\sum_{k=1}^{\infty}\left|\frac{w_{k}^{\prime}-w_{k}}{1-\bar{w}_{k} w_{k}^{\prime}}\right|$,$$
\sum_{k=1}^{\infty} \frac{\left|w_{k}^{\prime}-w_{k}\right|}{1-\left|w_{k}\right| \cdot\left|w_{k}^{\prime}\right|}
$$$$
\sum_{k=1}^{\infty} \frac{\left|w_{k}^{\prime}-w_{k}\right|}{1-\left|w_{k}\right|^{2}}
$$
(v) $\sum_{k=1}^{\infty} \frac{\left|w_{k}^{\prime}-w_{k}\right|}{1-\left|w_{k}^{\prime}\right|^{2}}$
(vi) $\sum_{k=1}^{\infty} \frac{\left|w_{k}^{\prime}-w_{k}\right|}{1-\left|w_{k}^{\prime}\right|}$.

$$
\begin{aligned}
& \sum_{k=1}^{\infty} \frac{\left|w_{k}^{\prime}-w_{k}\right|}{1-\left|w_{k}\right|}, \\
& \sum_{k=1}^{\infty} \frac{\left|w_{k}^{\prime}-w_{k}\right|}{1-\left|w_{k}^{\prime}\right|} .
\end{aligned}
$$


If the series converge and one of the sequences is a Blaschke sequence, then so is the other sequence.

The proof of this lemma is straightforward and will be omitted.

In the next theorem, the symbol $\mathcal{A}$ will be used for the disk algebra (consisting of all $C$-valued continuous functions on the closed unit disk which are analytic on the open disk), and $\mathcal{A}^{-1}$ will denote the set of invertible elements of $\mathcal{A}$ (the functions $f \in \mathcal{A}$ such that $\left.f^{-1} \in \mathcal{A}\right)$. The closed unit disk will be denoted by $\operatorname{cl} D$.

THEOREM 27. Let $\left(w_{k}\right)_{k=1}^{\infty}$ and $\left(w_{k}^{\prime}\right)_{k=1}^{\infty}$ be Blaschke sequences in $D$ such that the series (i)-(vi) in Lemma 26 are convergent. Then $\prod_{k=1}^{\infty}\left(1-\bar{w}_{k} z\right)\left(1-\bar{w}_{k}^{\prime} z\right)^{-1}$ converges absolutely and uniformly on $\mathrm{cl} D$. Let $m$ denote the function on $\mathrm{cl} D$ defined as the value of this product. Then:

(a) $m \in \mathcal{A}^{-1}$, and $m^{-1}=\prod_{k=1}^{\infty}\left(1-\bar{w}_{k}^{\prime} z\right)\left(1-\bar{w}_{k} z\right)^{-1}$.

(b) $1 / C \leq|m| \leq C^{\prime}$, where

$$
C=\exp \left\{\sum_{k=1}^{\infty} \frac{\left|w_{k}^{\prime}-w_{k}\right|}{1-\left|w_{k}\right|}\right\} \quad \text { and } \quad C^{\prime}=\exp \left\{\sum_{k=1}^{\infty} \frac{\left|w_{k}^{\prime}-w_{k}\right|}{1-\left|w_{k}^{\prime}\right|}\right\} \text {. }
$$

(c) $m$ is a multiplier from $\mathcal{H}(\phi)$ onto $\mathcal{H}(\psi)$, where $\phi$ and $\psi$ are the Blaschke products for the sequences $\left(w_{k}\right)$ and $\left(w_{k}^{\prime}\right): \phi=$ $\prod_{k=1}^{\infty} \beta_{w_{k}}$ and $\psi=\prod_{k=1}^{\infty} \beta_{w_{k}^{\prime}}$.

Proof. For each $z \in \mathrm{cl} D$ and $k \in N$,

$$
\left|\frac{1-\bar{w}_{k} z}{1-\bar{w}_{k}^{\prime} z}-1\right|=\left|z \cdot \frac{\bar{w}_{k}^{\prime}-\bar{w}_{k}}{1-\bar{w}_{k}^{\prime} z}\right| \leq \frac{\left|w_{k}^{\prime}-w_{k}\right|}{1-\left|w_{k}^{\prime}\right|} .
$$

By hypothesis, the series $\sum\left|w_{k}^{\prime}-w_{k}\right|\left(1-\left|w_{k}^{\prime}\right|\right)^{-1}$ converges. It follows that the product $\Pi\left(1-\bar{w}_{k} z\right)\left(1-\bar{w}_{k}^{\prime} z\right)^{-1}$ converges absolutely and uniformly on $\operatorname{cl} D$. Let $m$ be the function defined by this product. Then $m$ is continuous, and $m$ is analytic on $D$-that is, $m \in \mathcal{A}$.

For each $z \in \operatorname{cl} D$ and $k \in N$,

$$
\frac{1-\left|w_{k}\right|}{1+\left|w_{k}^{\prime}\right|} \leq\left|\frac{1-\bar{w}_{k} z}{1-\bar{w}_{k}^{\prime} z}\right| \leq \frac{1+\left|w_{k}\right|}{1-\left|w_{k}^{\prime}\right|} \text {. }
$$

Thus each factor in the product $\Pi\left(1-\bar{w}_{k} z\right)\left(1-\bar{w}_{k}^{\prime} z\right)^{-1}$ is bounded away from 0 and bounded away from $\infty$. For such products, uniform 
convergence means that the sequence of partial products converges uniformly to a limit which is also bounded away from 0 and $\infty$. Therefore $m \in \mathcal{A}^{-1}$, and in fact $m^{-1}$ is given by the product $\Pi(1-$ $\left.\bar{w}_{k}^{\prime} z\right)\left(1-\bar{w}_{k} z\right)^{-1}$.

Let $C$ and $C^{\prime}$ be the constants defined in the statement of the theorem. Both are finite, since they are defined in terms of convergent series. For each $n \in N$, let $m_{n}=\prod_{k=1}^{n}\left(1-\bar{w}_{k} z\right) /\left(1-\bar{w}_{k}^{\prime} z\right)$. Then, for each $n$ and for all $z \in \operatorname{cl} D$,

$$
\begin{aligned}
&\left|m_{n}(z)\right|=\prod_{k=1}^{n}\left|\frac{1-\bar{w}_{k} z}{1-\bar{w}_{k}^{\prime} z}\right| \\
&=\prod_{k=1}^{n}\left|1+z \cdot \frac{\bar{w}_{k}^{\prime}-\bar{w}_{k}}{1-\bar{w}_{k}^{\prime} z}\right| \\
& \leq \prod_{k=1}^{n}\left(1+\frac{\left|w_{k}^{\prime}-w_{k}\right|}{1-\left|w_{k}^{\prime}\right|}\right) \\
& \leq \prod_{k=1}^{n} \exp \left(\frac{\left|w_{k}^{\prime}-w_{k}\right|}{1-\left|w_{k}^{\prime}\right|}\right)=\exp \left(\sum_{k=1}^{n} \frac{\left|w_{k}^{\prime}-w_{k}\right|}{1-\left|w_{k}^{\prime}\right|}\right) \\
& \leq \exp \left(\sum_{k=1}^{\infty} \frac{\left|w_{k}^{\prime}-w_{k}\right|}{1-\left|w_{k}^{\prime}\right|}\right)
\end{aligned}
$$

Thus $\left|m_{n}\right| \leq C^{\prime}$. Similarly $\left|m_{n}^{-1}\right| \leq C$, that is, $\left|m_{n}\right| \geq 1 / C$. Letting $n \rightarrow \infty, 1 / C \leq|m| \leq C^{\prime}$.

It remains to prove (c). Let $f$ be any function in $\mathcal{H}(\phi)$. For $n \in N$, let $\phi_{n}=\prod_{k=1}^{n} \beta_{w_{k}}$ and $\psi_{n}=\prod_{k=1}^{n} \beta_{w_{k}^{\prime}}$. Note that $\mathcal{H}\left(\psi_{n}\right)=m_{n} \mathcal{H}\left(\phi_{n}\right)$ (Corollary 23). Let $f_{n}$ be the orthogonal projection of $f$ onto $\mathcal{H}\left(\phi_{n}\right)$. Since each function $\phi_{n}$ divides $\phi$ and the sequence $\left(\phi_{n}\right)_{n=1}^{\infty}$ converges pointwise to $\phi$, it can be shown that $\mathcal{H}(\phi)$ is the closed linear span of the subspaces $\mathcal{H}\left(\phi_{n}\right)[6]$ (pp. 34,35). Consequently $f_{n} \rightarrow f$ in $H^{2}$ as $n \rightarrow \infty$. Since the functions $m$ and $m_{n}$ are uniformly bounded (by $C^{\prime}$ ), $m_{n} f_{n} \rightarrow m f$ in $H^{2}$ as $n \rightarrow \infty$. Thus $m f$ is the limit of a sequence in $\mathcal{H}(\psi)$, and is therefore in $\mathcal{H}(\psi)$. This proves $m \mathcal{H}(\phi) \subseteq$ $\mathcal{H}(\psi)$. By the same argument, $m^{-1} \mathcal{H}(\psi) \subseteq \mathcal{H}(\phi)$. Thus $m \mathcal{H}(\phi)=$ $\mathcal{H}(\psi)$.

8. Application to the Annulus. The above results concerning multipliers between $S^{*}$-invariant subspaces in $H^{2}$ on the disk can 
be applied to the $Z$-invariant subspaces of $H^{2}$ on an annulus, where $Z$ denotes the operator of multiplication by $z$. These subspaces have been characterized by D. Hitt [3]. It was the application of multipliers in understanding Hitt's characterization which originally motivated their investigation.

In the interest of precision, the notation $L^{2}(T)$ will now be used in place of $L^{2}$, and the space $H^{2}$ will be written either as $H^{2}(T)$ (when viewed as a subspace of $L^{2}(T)$ ) or as $H^{2}(D)$ (when regarded as a space of analytic functions on $D$ ).

Let $r \in R^{+}$. Let $r D=\{r z: z \in D\}$ (that is, the disk $|z|<r$ ), and let $r T=\{r \zeta: \zeta \in T\}$ (that is, the circle $|z|=r$ ). Let $A$ denote the annulus bounded by the circles $T$ and $r T$. The Hilbert space $H^{2}(\partial A)$ is defined to be a subspace of $L^{2}(T) \oplus L^{2}(r T)$ as follows:

$$
H^{2}(\partial A)=\left\{(f, g) \in L^{2}(T) \oplus L^{2}(r T): \hat{g}(n)=r^{n} \hat{f}(n) \text { for all } n \in Z\right\}
$$

where $\hat{f}$ denotes the Fourier transform of $f$. For each function $(f, g)$ in $H^{2}(\partial A)$, there is an analytic function on $A$ with $(f, g)$ as its boundary value function. These analytic functions form a space $H^{2}(A)$. The norm of a function in $H^{2}(A)$ is defined to be the norm of its boundary value function in $H^{2}(\partial A)$. Thus the spaces $H^{2}(A)$ and $H^{2}(\partial A)$ are isometrically isomorphic, like $H^{2}(D)$ and $H^{2}(T)$.

An inner function on $A$ is a function $(f, g)$ in $H^{\infty}(\partial A)$ such that $(|f|,|g|)=\left(a_{1}, a_{2}\right)$ for some positive constants $a_{1}$ and $a_{2}$ (depending on $f$ and $g$ ). Divisibility can be defined for inner functions on $A$ just as on $D$ : if $\Phi$ and $\Psi$ are inner functions, we say that $\Psi$ divides $\Phi$ when there is an inner function $\Xi$ such that $\Phi=\Psi \Xi$. There is also a definition of outer functions on $A$, resulting in a factorization theorem as follows: each function $f \in H^{2}(A)$ can be factored as the product of an inner function and an outer function, and this factorization is unique up to multiplication by functions of the form $c z^{n}$, where $c \in C \backslash\{0\}$ and $n \in Z$ (see [3]). These functions $c z^{n}$ are called units. They are inner functions which divide every inner function, and they are also outer functions.

Assume $r<1$. Let $D^{-1}=\left\{z^{-1}: z \in D\right\}$, and let $H_{0}^{2}\left(r D^{-1}\right)=$ $z^{-1} H^{2}\left(r D^{-1}\right)$. If $f \in H^{2}(D)$ and $g \in H_{0}^{2}\left(r D^{-1}\right)$, then $f+g$ is defined and analytic on $A$-in fact, $f+g \in H^{2}(A)$. If $h \in H^{2}(A)$, then the Laurent series for $h$ provides a decomposition of $h$ in the form $h=f+g$, where $f \in H^{2}(D)$ and $g \in H_{0}^{2}\left(r D^{-1}\right)$. What has 
just been described is a one-to-one correspondence between $H^{2}(A)$ and $H^{2}(D) \oplus H_{0}^{2}\left(r D^{-1}\right)$. This correspondence is a topological linear isomorphism but not an isometry.

Let $r \in(0,1)$, and let $A=\{z \in C: r<|z|<1\}$. If $F \in$ $H^{2}(A)$ has boundary value function $(f, g) \in H^{2}(\partial A)$, then $z F$ has boundary value function $(z f, z g)$. Then $z F \in H^{2}(A)$, and $\|z F\|_{A}^{2}=$ $\|f\|^{2}+r\|g\|^{2} \leq\|f\|^{2}+\|g\|^{2}=\|F\|_{A}^{2}$, where $\|\cdot\|_{A}$ denotes the norm in $H^{2}(\partial A)$. Hence multiplication by $z$ is a bounded linear operator on $H^{2}(A)$, which we denote by $Z$. This operator is invertible-its inverse is multiplication by $z^{-1}$.

If $\Phi$ is an inner function on $A$, then $\Phi H^{2}(A)$ is a subspace of $H^{2}(A)$ which is both $Z$-invariant and $Z^{-1}$-invariant. Conversely, if $\mathcal{M}$ is a subspace of $H^{2}(A)$ which is both $Z$-invariant and $Z^{-1}$ invariant, and if $\mathcal{M} \neq\{0\}$, then $\mathcal{M}=\Phi H^{2}(A)$ for some inner function $\Phi$. (For a proof of this, see [8, Theorem 1].) These subspaces are called fully invariant subspaces of $H^{2}(A)$. Subspaces which are $Z^{-1}$-invariant but not $Z$-invariant are called simply $Z^{-1}$-invariant.

The simply $Z^{-1}$-invariant subspaces of $H^{2}(A)$ have been characterized by $\mathrm{D}$. Hitt [3]. Actually Hitt's annulus is $A=\{z \in C$ : $1<|z|<R\}$, where $R>1$, and his characterization concerns the $Z$-invariant subspaces of $H^{2}(A)$ for this annulus. However the transformation $z \mapsto 1 / z$ yields an equivalent characterization of the $Z^{-1}$-invariant subspaces for our annulus.

Hitt's characterization involves subspaces $\mathcal{M}_{\phi}$ defined as follows: if $\phi$ is any inner function on $D, \mathcal{M}_{\phi}=\mathcal{H}(\phi) \oplus H_{0}^{2}\left(r D^{-1}\right)$. When $H^{2}(A)$ is identified with $H^{2}(D) \oplus H_{0}^{2}\left(r D^{-1}\right)$, the operator $Z^{-1}$ acts as follows:

$$
Z^{-1}(f+g)=S^{*} f+z^{-1}[g+f(0)] \quad\left(f \in H^{2}(D), g \in H_{0}^{2}\left(r D^{-1}\right)\right) .
$$

Consequently, for any inner function $\phi, \mathcal{M}_{\phi}$ is a simply $Z^{-1}$-invariant subspace. According to Hitt, if $\mathcal{M}$ is any simply $Z^{-1}$-invariant subspace of $H^{2}(A)$, then $\mathcal{M}=\Phi f \mathcal{M}_{\phi}$ for some $\phi, f$ and $\Phi$, where $\phi$ is an inner function on $D, \Phi$ is an inner function on $A$, and $f$ is an outer function in $H^{2}(D)$ such that multiplication by $f$ is isometric on $\mathcal{H}(\phi)$ in $H^{2}(D)$. The functions $\Phi, f$ and $\phi$ are not uniquely determined by $\mathcal{M}$. The inner function $\Phi$ is the greatest common divisor of the inner factors of the functions in $\mathcal{M}$, and is determined uniquely up to multiplication by units. The following propositions describe the non-uniqueness of $f$ and $\phi$. 
Proposition 28. Let $\phi$ and $\psi$ be inner functions on $D$, and let $m \in H^{\infty}(D)$ such that $m^{-1} \in H^{\infty}(D)$. Then $m \mathcal{H}(\phi)=\mathcal{H}(\psi)$ if and only if $m \mathcal{M}_{\phi}=\mathcal{M}_{\psi}$.

Proof. According to the definitions above, $\mathcal{M}_{\phi}=\mathcal{H}(\phi) \oplus H_{0}^{2}\left(r D^{-1}\right)$ and $\mathcal{M}_{\psi}=\mathcal{H}(\psi) \oplus H_{0}^{2}\left(r D^{-1}\right)$. Suppose $m \mathcal{M}_{\phi}=\mathcal{M}_{\psi}$. Then certainly $m \mathcal{H}(\phi) \subseteq \mathcal{M}_{\psi}$, and since $m$ is analytic on $D$, it follows that $m \mathcal{H}(\phi) \subseteq \mathcal{H}(\psi)$. Similarly, $m^{-1} \mathcal{H}(\psi) \subseteq \mathcal{H}(\phi)$, and thus $m \mathcal{H}(\phi)=\mathcal{H}(\psi)$.

Now suppose $m \mathcal{H}(\phi)=\mathcal{H}(\psi)$. To prove $m \mathcal{M}_{\phi} \subseteq \mathcal{M}_{\psi}$, it will suffice to show that $m H_{0}^{2}\left(r D^{-1}\right) \subseteq \mathcal{M}_{\psi}$. Note that $H_{0}^{2}\left(r D^{-1}\right)$ is the closed linear span in $H^{2}(A)$ of the set $\left\{z^{-j}: j \in N\right\}$. Since $m$ is bounded on $A$, multiplication by $m$ is continuous on $H^{2}(A)$. Consequently $m H_{0}^{2}\left(r D^{-1}\right) \subseteq \mathcal{M}_{\psi}$ if and only if $m z^{-j} \in \mathcal{M}_{\psi}$ for each $j \in N$. For $j=1, m z^{-1}=S^{*} m+m(0) z^{-1}$, and this is in $\mathcal{M}_{\psi}$ because $S^{*} m \in \mathcal{H}(\psi)$ (Corollary 3). By induction, $m z^{-j}=$ $S^{* j} m+g_{j}$, where $g_{j} \in H_{0}^{2}\left(r D^{-1}\right)$, and hence $m z^{-j} \in \mathcal{M}_{\psi}$. This completes the proof that $m \mathcal{M}_{\phi} \subseteq \mathcal{M}_{\psi}$. By the same argument, $m^{-1} \mathcal{M}_{\psi} \subseteq \mathcal{M}_{\phi}$, and thus $m \mathcal{M}_{\phi}=\mathcal{M}_{\psi}$.

Proposition 29. Let $\phi_{1}$ and $\phi_{2}$ be inner functions on $D$. Let $f_{1}$ and $f_{2}$ be outer functions on $D$ such that $f_{1}$ acts isometrically when multiplying $\mathcal{H}\left(\phi_{1}\right)$ and $f_{2}$ acts isometrically when multiplying $\mathcal{H}\left(\phi_{2}\right)$. If $f_{1} \mathcal{M}_{\phi_{1}}=f_{2} \mathcal{M}_{\phi_{2}}$, then $f_{1} / f_{2}$ is an isometric multiplier from $\mathcal{H}\left(\phi_{1}\right)$ onto $\mathcal{H}\left(\phi_{2}\right)$, and there exists $w \in D$ such that $\phi_{2}=$ $\alpha_{w} \circ \phi_{1}$, where $\alpha_{w}=(w-z) /(1-\bar{w} z)$.

Proof. If $f_{1} \mathcal{M}_{\phi_{1}}=f_{2} \mathcal{M}_{\phi_{2}}$, then $\left(f_{1} / f_{2}\right) \mathcal{H}\left(\phi_{1}\right)=\mathcal{H}\left(\phi_{2}\right)$, and $f_{1} / f_{2}$ multiplies $\mathcal{H}\left(\phi_{1}\right)$ isometrically. This means that $f_{1} / f_{2}$ is an isometric multiplier from $\mathcal{H}\left(\phi_{1}\right)$ onto $\mathcal{H}\left(\phi_{2}\right)$. By Theorem 10, $\phi_{2}=\alpha_{w} \circ \phi_{1}$ for some $w \in D$.

\section{Open questions.}

1. Are there any unbounded multipliers? More precisely, if $m$ is a multiplier between subspaces $\mathcal{H}(\phi)$ and $\mathcal{H}(\psi)$, where $\phi$ and $\psi$ are inner functions, is it necessary that $m \in H^{\infty}$ ?

2. Let $\mathcal{I}$ denote the set of inner functions with the topology inherited from $H^{\infty}$. The connected components of $\mathcal{I}$ have been 
studied by $V$. Nestoridis $[4,5]$, among others. If there exists a multiplier between subspaces $\mathcal{H}(\phi)$ and $\mathcal{H}(\psi)$, is it true that the inner functions $\phi$ and $\psi$ must be in the same component? Based on the theorems and examples above, this would appear to be a reasonable conjecture. The converse is false, however. There are nonconstant inner functions $\phi$ such that $\phi$ and $z \phi$ can be joined by a path in $\mathcal{I}$ [4] (p. 475, Proposition 2), whereas there is no multiplier between $\mathcal{H}(\phi)$ and $\mathcal{H}(z \phi)$ (Corollary 21).

Acknowledgements. The work presented above is the essence of the author's doctoral dissertation, completed in 1991 at the University of California, Berkeley, under the direction of Professor Donald Sarason. The author is grateful to Professor Sarason for numerous helpful discussions. The ideas for Theorem 14 and its proof are his.

\section{REFERENCES}

[1] L. de Branges and J. Rovnyak, Square Summable Power Series, Holt, Rinehart and Winston, New York, 1966.

[2] R. G. Douglas, On Majorization, Factorization, and Range Inclusion of Operators on Hilbert Space, Proc. Amer. Math. Soc., 17 (1966), 413415.

[3] D. Hitt, Invariant Subspaces of $H^{2}$ of an Annulus, Pacific J. Math., 134 (1) (1988), 101-120.

[4] V. Nestoridis, Inner Functions: Invariant Connected Components, Pacific J. Math., 83 (2)(1979), 473-480.

[5] V. Nestoridis, Inner Functions: Noninvariant Connected Components, Pacific J. Math., 87 (1) (1980), 199-209.

[6] N. K. Nikol'skiı̌, Treatise on the Shift Operator, Grundlehren der mathematischen Wissenshaften, 273, Springer-Verlag, New York, 1986.

[7] R. G. Douglas, H. S. Shapiro and A. L. Shields, Cyclic Vectors and Invariant Subspaces for the Backward Shift Operator, Annales de l'Institut Fourier (Grenoble), 20 (1) (1970), 37-76.

[8] H. L. Royden, Invariant Subspaces of $\mathcal{H}^{p}$ for Multiply Connected Regions, Pacific J. Math., 134 (1) (1988), 151-172.

[9] D. Sarason, Shift-invariant Spaces from the Brangesian Point of View, in The Bieberbach Conjecture-Proceedings of the Symposium on the Occasion of the Proof, Amer. Math. Soc., Providence, 1986, 153-166. 
[10] D. Sarason, Doubly Shift-invariant spaces in $H^{2}$, J. Operator Theory, 16 (1986), 75-97.

[11] D. Sarason, Nearly Invariant Subspaces of the Backward Shift, Operator Theory: Adv. Appl., 35 (1988), 481-493.

Received July 15, 1992, revised December 15, 1992 and accepted for publication December 21, 1992.

University College of the Cariboo Kamloops, B.C. CANAdA 



\section{PACIFIC JOURNAL OF MATHEMATICS}

Volume $166 \quad$ No. $2 \quad$ December 1994

Geometric aspects of Bäcklund transformations of Weingarten

submanifolds

STEVEN BUYSKE

Multipliers between invariant subspaces of the backward shift

225

ROBERT BRUCE CROFOOT

The Cauchy integral, analytic capacity and subsets of quasicircles

XIANG FANG

The number of lattice points within a contour and visible from the origin 295

Douglas Austin Hensley

On flatness of the Coxeter graph $E_{8}$

305

MASAKI IZUMI

Immersions up to joint-bordism

GUI SONG LI

Generalization of the Hilbert metric to the space of positive definite matrices

CARlangelo Liverani and Maciej WojtKowski

Periodicity, genera and Alexander polynomials of knots

SWATEE NAIK

On divisors of sums of integers. $\mathrm{V}$

ANDRÁS SÁRKÖZY and CAMERON LEIGH STEWART

Approximately inner automorphisms on inclusions of type $\mathrm{III}_{\lambda}$-factors

CARL WINSLøW

Correction to: "A convexity theorem for semisimple symmetric spaces"

KARL-HERMANN NEEB

Correction to: "Periodic points on nilmanifolds and solvmanifolds"

EDWARD KEPPELMANN

Correction to: "Partially measurable sets in measure spaces" 